\title{
Arc-transitive dihedral regular covers of cubic graphs
}

\author{
Jicheng $\mathrm{Ma}^{*}$ \\ Department of Mathematics \& KLDAIP \\ Chongqing University of Arts and Sciences \\ Chongqing 402160, China \\ ma_jicheng@hotmail.com
}

Submitted: Jan 16, 2014; Accepted: Jun 30, 2014; Published: Jul 10, 2014

Mathematics Subject Classifications: 05C25, 20B25

\begin{abstract}
A regular covering projection is called dihedral or abelian if the covering transformation group is dihedral or abelian. A lot of work has been done with regard to the classification of arc-transitive abelian (or elementary abelian, or cyclic) covers of symmetric graphs. In this paper, we investigate arc-transitive dihedral regular covers of symmetric (arc-transitive) cubic graphs. In particular, we classify all arc-transitive dihedral regular covers of $K_{4}, K_{3,3}$, the 3 -cube $Q_{3}$ and the Petersen graph.
\end{abstract}

Keywords: Arc-transitive graph; Regular cover; Dihedral cover; Cubic graph

\section{Introduction}

Covering techniques are known to be a useful tool in algebraic and topological graph theory. Application of these techniques has resulted in many important examples and classification of certain families of graphs with particular symmetry properties. For example, Djoković used graph covers to prove that there exist infinitely many 5-arc-transitive cubic graphs, as elementary abelian covers of Tutte's 8-cage.

Recently, quite a lot of attention has been paid to the classification of arc-transitive covers of symmetric graphs. Approaches have involved voltage graph techniques (see [9]) and universal group methods (see [3]). In most cases, the group of covering transformation is either cyclic or elementary abelian, or more generally abelian. These methods have been

*Supported by CUAS grant R2012SC22 and Program for Innovation Team Building at Institutions of Higher Education in Chongqing (grant no. KJTD201321) 
successfully applied in the classification of arc-transitive elementary abelian or abelian covers of symmetric cubic graphs, such as the complete graph $K_{4}$, the complete bipartite graph $K_{3,3}$, the 3 -cube graph $Q_{3}$, the Petersen graph and the Heawood graph and so on.

In this paper, we are aiming to extend our research on arc-transitive abelian covers to non-abelian covers which is harder and has not been previously considered. We begin with some further background in Section 2, and determine the arc-transitive cyclic regular covers of the Möbius-Kantor graph and the Desargues graph in Sections 3 and 4, respectively. In Section 5, we deal with dihedral covers, and give a complete classification of arc-transitive dihedral covers of $K_{4}, K_{3,3}, Q_{3}$ and the Petersen graph.

\section{Preliminaries}

Throughout this paper, all the graphs are finite and simple. A covering projection is defined as a graph homomorphism $p: \tilde{X} \rightarrow X$ which is surjective and locally bijective, which means that the restriction $p: N(\tilde{v}) \rightarrow N(v)$ is a bijection whenever $\tilde{v}$ is a vertex of $\tilde{X}$ such that $p(\tilde{v})=v \in V(X)$. We call $X$ the base graph, $\tilde{X}$ a covering graph. A covering projection $p: \tilde{X} \rightarrow X$ is called regular if there exists a semi-regular subgroup $N$ of the automorphism group $\operatorname{Aut}(\tilde{X})$ of $\tilde{X}$ such that the quotient graph $\tilde{X} / N$ (with vertices taken as the orbits of $N$, and two vertices adjacent whenever there exists an edge between these two $N$-orbits) is isomorphic to $X$. In that case we call $\tilde{X}$ a regular cover of $X$. The regular covering projection is called dihedral (or cyclic) if $N$ is a dihedral (or cyclic) group. Similarly, we say a regular covering projection is abelian (or elementary abelian) when the group $N$ is abelian (or elementary abelian).

Let $p: \tilde{X} \rightarrow X$ be a covering projection, and suppose $\alpha$ and $\beta$ are automorphisms of $X$ and $\tilde{X}$ such that $\alpha \circ p=p \circ \beta$, that is, such that the following diagram commutes:

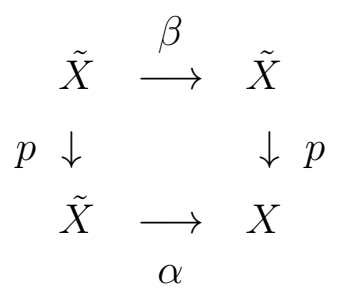

Then we say that $\alpha$ lifts along $p$ to $\beta$, and $\beta$ projects to $\alpha$, and also we call $\beta$ a lift of $\alpha$, and $\alpha$ a projection of $\beta$. Note that $\alpha$ is uniquely determined by $\beta$, but $\beta$ is not generally determined by $\alpha$. The set of all lifts of a given $\alpha \in \operatorname{Aut}(X)$ is denoted by $\tilde{\alpha}$. If every automorphism of a subgroup $G$ of $\operatorname{Aut}(X)$ lifts, then $\bigcup_{\alpha \in G} \tilde{\alpha}$ is a subgroup of $\operatorname{Aut}(\tilde{X})$, called the lift of $G$.

In particular, the lift of the identity subgroup of $\operatorname{Aut}(X)$ (or equivalently, the subgroup of all automorphisms of $\tilde{X}$ that project to the identity automorphism of $X$ ) is called the group of covering transformations, or voltage group, and is sometimes denoted by $\mathrm{CT}(p)$. The normalizer of $\mathrm{CT}(p)$ in $\operatorname{Aut}(\tilde{X})$ projects to the largest subgroup of $\operatorname{Aut}(X)$ that lifts. Hence in particular, if the latter subgroup is $A$, say, then the lift of $A$ has a normal subgroup $\mathrm{CT}(p)$ with quotient isomorphic to $A$. 
Two regular covering projections $p: Y \rightarrow X$ and $p^{\prime}: Y^{\prime} \rightarrow X$ are called isomorphic if there exist graph isomorphism $\tilde{\theta}: Y \rightarrow Y^{\prime}$ and graph automorphism $\theta: X \rightarrow X$ such that $\theta p=p^{\prime} \tilde{\theta}$. In particular, isomorphic covering projections $p$ and $p^{\prime}$ are called equivalent, if $\theta$ is the trivial automorphism. Similarly, two regular covers $Y$ and $Y^{\prime}$ are called equivalent if the two regular covering projections $p$ and $p^{\prime}$ are equivalent. Usually, regular covers are studied up to equivalence.

For every symmetric cubic graph, we now know that the automorphism group is a quotient of one of seven finitely-presented groups, which can be listed as $G_{1}, G_{2}^{1}, G_{2}^{2}, G_{3}$, $G_{4}^{1}, G_{4}^{2}$ and $G_{5}$, and presented as follows (see $[5,4]$ )

$$
\begin{aligned}
& G_{1}=\left\langle h, a \mid h^{3}=a^{2}=1\right\rangle ; \\
& G_{2}^{1}=\left\langle h, p, a \mid h^{3}=p^{2}=a^{2}=1, p h p=h^{-1}, a^{-1} p a=p\right\rangle ; \\
& G_{2}^{2}=\left\langle h, p, a \mid h^{3}=p^{2}=1, a^{2}=p, p h p=h^{-1}, a^{-1} p a=p\right\rangle ; \\
& G_{3}=\left\langle h, p, q, a \mid h^{3}=p^{2}=q^{2}=a^{2}=1, p q=q p, p h p=h, q h q=h^{-1}, a^{-1} p a=q\right\rangle ; \\
& G_{4}^{1}=\langle h, p, q, r, a| h^{3}=p^{2}=q^{2}=r^{2}=a^{2}=1, p q=q p, p r=r p, \quad(q r)^{2}=p, \\
& \left.h^{-1} p h=q, h^{-1} q h=p q, r h r=h^{-1}, a^{-1} p a=p, a^{-1} q a=r\right\rangle ; \\
& G_{4}^{2}=\langle h, p, q, r, a| h^{3}=p^{2}=q^{2}=r^{2}=1, a^{2}=p, p q=q p, p r=r p, \quad(q r)^{2}=p, \\
& \left.h^{-1} p h=q, h^{-1} q h=p q, r h r=h^{-1}, a^{-1} p a=p, a^{-1} q a=r\right\rangle ; \\
& G_{5}=\langle h, p, q, r, s, a| h^{3}=p^{2}=q^{2}=r^{2}=s^{2}=a^{2}=1, p q=q p, p r=r p, p s=s p, \\
& q^{2}=r q, q s=s q,(r s)^{2}=p q, h^{-1} p h=p, h^{-1} q h=r, \\
& \left.h^{-1} r h=p q r, s h s=h^{-1}, a^{-1} p a=q, a^{-1} r a=s\right\rangle .
\end{aligned}
$$

If a finite group $G$ acts as an $s$-arc-regular group of automorphisms of a cubic graph $X$, then $G$ is a smooth quotient of $G_{s}$ or $G_{s}^{i}$, where $i=1$ or 2 depending on whether or not the group contains an involution $a$ that reverses an arc (in the cases where $s$ is even). (Note, 'smooth' here means that the orders of the generators are preserved.) Let $\mathcal{U}$ be either $G_{s}$ or $G_{s}^{i}$, then $G$ is a smooth quotient $\mathcal{U} / K$ of $\mathcal{U}$ by some torsion-free normal subgroup $K$. If $\tilde{X}$ is a regular cover of $X$ admitting a group action of the same type, then there exists a normal subgroup $L$ of $\mathcal{U}$ contained in $K$, with $\mathcal{U} / L$ being the corresponding group automorphisms of $\tilde{X}$. Then the group $\mathcal{U} / L$ is an extension of the covering group $K / L$ by the given group $G=\mathcal{U} / K$. In order to find all cyclic covers, we need to find all possibilities for $L$ such that $K / L$ is cyclic. The presentation of $K$ can be found using Reidemeister-Schreier Theory, or by use of the Rewrite command in MAgma [1]. In the cases we will consider, $K$ is a free abelian group of finite rank $d$, namely the Betti number of the base graph $X$, with some basis $\left\{w_{1}, w_{2}, \cdots, w_{d}\right\}$. Algebraic or computational techniques can be applied to find the actions by conjugation of the generators of $\mathcal{U}$ on the generators of $K$. And these actions induce linear transformations of the free abelian group $K$. Equivalently, a $d$-dimensional matrix representation of the group $G=\mathcal{U} / K$ can be given. Therefore, in order to find all the cyclic covers, we need to find all the $G$ invariant subgroups $L$ of rank $d-1$, equivalently we need to find all the $(d-1)$-dimensional representation of $G$. 
More details of Conder and the author's universal group method can be seen in [3] and [8]. Here we introduce some computational techniques that involves using MAGma. To find all finite cyclic regular covers with cyclic covering groups of exponent $m$, we may consider the action of $G$ by conjugation on the generators of $K / K^{(m)}$, where $K^{(m)}$ is the characteristic subgroup of $K$ generated by the $m$ th powers of all elements of $K$. Since $K / K^{(m)}$ is $G$-invariant, we use MAGMA to construct a finite group $K / K^{(m)} \rtimes G$ which is the extension group of $G$ by $K / K^{(m)}$. Note that this can be done, since both $K / K^{(m)}$ and $G$ are finitely-presented subgroups of $\mathcal{U}$. With a finite group stored in MAGMA we can use the commands NormalSubgroups and meet to find all the subgroups $L$ of $K / K^{(m)}$ which are normal in $K / K^{(m)} \rtimes G$. Note that the 'type' of group $K / K^{(m)} \rtimes G$ may not work for using the above commands in MAGMA, then what one needs to do is using the double coset graph construction method (more details can be seen in [3, Section 2]) to transform it into a permutation group (namely, an arc-transitive group of automorphisms). This method works successfully for 'small' integer $m$. Generally, if $m=p_{1}^{e_{1}} p_{2}^{e_{2}} \cdots p_{t}^{e_{t}}$ is the prime-power factorisation of $m$ with distinct primes $p_{i}$, then the factor $K / L$ is a direct product of its Sylow subgroups. It follows that we need only consider the $G$-invariant subgroups of prime-power index in $K / K^{(m)}$.

Once all the possibilities for $L$ have been found, we can determine additional information, such as uniqueness up to isomorphism and arc-transitivity of the covering graphs.

\section{Arc-transitive cyclic regular covers of the Möbius- Kantor graph}

In this section, we classify all the arc-transitive cyclic covering graphs of the MöbiusKantor graph $G P(8,3)$. The automorphism group of $G P(8,3)$ is isomorphic to $\operatorname{GL}(2,3) \rtimes$ $\mathrm{C}_{2}$ and acts 2-arc-regularly on the arcs. There are two other 1-arc-regular subgroups $\mathrm{GL}(2,3)$ and $\mathrm{SL}(2,3) \rtimes C_{2}$.

Take the group $G_{2}^{1}$, with presentation $G=\left\langle h, a, p \mid h^{3}=p^{2}=a^{2}=(p h)^{2}=[a, p]=1\right\rangle$. The group $G_{2}^{1}$ has two normal subgroups of index 96 , both with quotient GL $(2,3) \rtimes C_{2}$, but these are interchanged by the outer automorphism that takes the three generators $h, a$ and $p$ to $h, a p$ and $p$ respectively, so without loss of generality we can take either one of them.

We will take the one that is contained in the subgroup $G_{1}=\langle h, a\rangle$; this is a normal subgroup $N$ of index 48 in $G_{1}$ with $G_{1} / N \cong \operatorname{GL}(2,3)$.

Using the Rewrite command in MAGMA, we find that the subgroup $N$ is free of rank 9 , on generators

$$
\begin{array}{ll}
w_{1}=\left(h^{-1} a h a h a\right)^{2} & w_{2}=\left(h^{-1} a h a h^{-1} a\right)^{2} \\
w_{3}=\left(h^{-1} a h^{-1} a h a\right)^{2} & w_{4}=\left(h a h a h^{-1} a\right)^{2} \\
w_{5}=\left(h a h^{-1} a h a\right)^{2} & w_{6}=\left(h a h^{-1} a h^{-1} a\right)^{2} \\
w_{7}=h^{-1} a h^{-1} a h^{-1} a h a h^{-1} a h^{-1} a h^{-1} & w_{8}=a h a h a h a h a h^{-1} a h^{-1} a h^{-1} a h^{-1} \\
w_{9}=a h^{-1} a h^{-1} a h^{-1} a h a h^{-1} a h^{-1} a h^{-1} a &
\end{array}
$$


Easy calculations show that the generators $h, a$ and $p$ act by conjugation as below: (Note that the actions of generator $a p$ is just the composition of $a$ and $p$.)

$$
\begin{array}{lll}
w_{1}^{a}=w_{3}{ }^{-1} & w_{1}{ }^{h}=w_{7}{ }^{-1} & w_{1}{ }^{p}=w_{6} \\
w_{2}{ }^{a}=w_{5}{ }^{-1} & w_{2}{ }^{h}=w_{2}{ }^{-1} w_{4} & w_{2}{ }^{p}=w_{5} \\
w_{3}{ }^{a}=w_{1}{ }^{-1} & w_{3}{ }^{h}=w_{3}{ }^{-1} w_{5} & w_{3}{ }^{p}=w_{4} \\
w_{4}{ }^{a}=w_{6}{ }^{-1} & w_{4}{ }^{h}=w_{2}{ }^{-1} & w_{4}{ }^{p}=w_{3} \\
w_{5}{ }^{a}=w_{2}{ }^{-1} & w_{5}{ }^{h}=w_{3}{ }^{-1} & w_{5}{ }^{p}=w_{2} \\
w_{6}{ }^{a}=w_{4}{ }^{-1} & w_{6}{ }^{h}=w_{1}{ }^{-1} & w_{6}{ }^{p}=w_{1} \\
w_{7}^{a}=w_{9} & w_{7}{ }^{h}=w_{6} & w_{7}{ }^{-1} \\
w_{8}{ }^{a}=w_{8}{ }^{-1} & w_{8}{ }^{h}=w_{1} w_{9} & w_{8}{ }^{p}=w_{7}{ }^{-1} w_{8} w_{9} \\
w_{9}{ }^{a}=w_{7} & w_{9}{ }^{h}=w_{1}{ }^{-1} w_{7} w_{8}{ }^{-1} w_{9}{ }^{-1} & w_{9}{ }^{p}=w_{9}{ }^{-1}
\end{array}
$$

Now take the quotient $G_{1} / N^{\prime}$ where $N^{\prime}$ is the derived subgroup of $N$, which is an extension of the free abelian group $N / N^{\prime} \cong \mathbb{Z}^{9}$ by the group $G_{1} / N \cong \operatorname{GL}(2,3)$, and replace the generators $h, a$ and all $w_{i}$ by their images in this group. Also let $K$ denote the subgroup $N / N^{\prime}$, and let $G$ be $G_{1} / N^{\prime}$. Then, in particular, $G$ is an extension of GL $(2,3)$ by $\mathbb{Z}^{9}$.

By the above observations, we see that the generators $h, a$ and $p$ induce linear transformations of the free abelian group $K \cong \mathbb{Z}^{9}$ as follows:

$$
\begin{array}{r}
a \mapsto\left(\begin{array}{rrrrrrrrr}
0 & 0 & -1 & 0 & 0 & 0 & 0 & 0 & 0 \\
0 & 0 & 0 & 0 & -1 & 0 & 0 & 0 & 0 \\
-1 & 0 & 0 & 0 & 0 & 0 & 0 & 0 & 0 \\
0 & 0 & 0 & 0 & 0 & -1 & 0 & 0 & 0 \\
0 & -1 & 0 & 0 & 0 & 0 & 0 & 0 & 0 \\
0 & 0 & 0 & -1 & 0 & 0 & 0 & 0 & 0 \\
0 & 0 & 0 & 0 & 0 & 0 & 0 & 0 & 1 \\
0 & 0 & 0 & 0 & 0 & 0 & 0 & -1 & 0 \\
0 & 0 & 0 & 0 & 0 & 0 & 1 & 0 & 0
\end{array}\right), \\
h \mapsto\left(\begin{array}{rrrrrrrrr}
0 & 0 & 0 & 0 & 0 & 0 & -1 & 0 & 0 \\
0 & -1 & 0 & 1 & 0 & 0 & 0 & 0 & 0 \\
0 & 0 & -1 & 0 & 1 & 0 & 0 & 0 & 0 \\
0 & -1 & 0 & 0 & 0 & 0 & 0 & 0 & 0 \\
0 & 0 & -1 & 0 & 0 & 0 & 0 & 0 & 0 \\
-1 & 0 & 0 & 0 & 0 & 0 & 0 & 0 & 0 \\
0 & 0 & 0 & 0 & 0 & 1 & 0 & 0 & 0 \\
1 & 0 & 0 & 0 & 0 & 0 & 0 & 0 & 1 \\
-1 & 0 & 0 & 0 & 0 & 0 & 1 & -1 & -1
\end{array}\right),
\end{array}
$$


and

$$
p \mapsto\left(\begin{array}{rrrrrrrrr}
0 & 0 & 0 & 0 & 0 & 1 & 0 & 0 & 0 \\
0 & 0 & 0 & 0 & 1 & 0 & 0 & 0 & 0 \\
0 & 0 & 0 & 1 & 0 & 0 & 0 & 0 & 0 \\
0 & 0 & 1 & 0 & 0 & 0 & 0 & 0 & 0 \\
0 & 1 & 0 & 0 & 0 & 0 & 0 & 0 & 0 \\
1 & 0 & 0 & 0 & 0 & 0 & 0 & 0 & 0 \\
0 & 0 & 0 & 0 & 0 & 0 & -1 & 0 & 0 \\
0 & 0 & 0 & 0 & 0 & 0 & -1 & 1 & 1 \\
0 & 0 & 0 & 0 & 0 & 0 & 0 & 0 & -1
\end{array}\right) .
$$

These matrices generate a group isomorphic to $\operatorname{Aut}(G P(8,3))$, with the first two generating a subgroup isomorphic to $\mathrm{GL}(2,3)$; and the first and the product of the first and the third generating a subgroup isomorphic to $\mathrm{SL}(2,3) \rtimes C_{2}$. Note that the matrices of orders 3,2 and 8 representing $h, a$ and $h a$ have traces $-3,-1$ and 1 , respectively.

Next, the character table of the group $\operatorname{GL}(2,3)$ is given in Table 1 , with $\gamma$ being the zeroes of the polynomial $t^{2}+2 t+3$.

Table 1: The character table of the group GL $(2,3)$

\begin{tabular}{crrrrrrrr}
\hline Element order & 1 & 2 & 2 & 3 & 4 & 6 & 8 & 8 \\
Class size & 1 & 1 & 12 & 8 & 6 & 8 & 6 & 6 \\
\hline$\chi_{1}$ & 1 & 1 & 1 & 1 & 1 & 1 & 1 & 1 \\
$\chi_{2}$ & 1 & 1 & -1 & 1 & 1 & 1 & -1 & -1 \\
$\chi_{3}$ & 2 & 2 & 0 & -1 & 2 & -1 & 0 & 0 \\
$\chi_{4}$ & 2 & -2 & 0 & -1 & 0 & 1 & $\gamma$ & $-\gamma$ \\
$\chi_{5}$ & 2 & -2 & 0 & -1 & 0 & 1 & $-\gamma$ & $\gamma$ \\
$\chi_{6}$ & 3 & 3 & 1 & 0 & -1 & 0 & -1 & -1 \\
$\chi_{7}$ & 3 & 3 & -1 & 0 & -1 & 0 & 1 & 1 \\
$\chi_{6}$ & 4 & -4 & 0 & 1 & 0 & -1 & 0 & 0 \\
\hline
\end{tabular}

By inspecting traces, we see that the character of the 9-dimensional representation of $\operatorname{GL}(2,3)$ over $\mathbb{Q}$ associated with the above action of $G=\langle h, a\rangle$ on $K$ is the character $\chi_{3}+\chi_{4}+\chi_{5}+\chi_{7}$, which is reducible to the sum of $\chi_{3}, \chi_{4}+\chi_{5}$ and $\chi_{7}$, which are characters of three irreducible 2-, 4- and 3-dimensional representations over the rational field $\mathbb{Q}$. Especially, the 4-dimensional representation is reducible to two 2-dimensional irreducible representations over fields containing zeroes of the polynomial $t^{2}+2 t+3$. Therefore, for any prime $k$ other than 2 and 3 , there is no $G$-invariant subgroup of rank 8. 
For prime integers 2 and 3, with the help of MAGMA by using the commands GModule and Submodules for matrix groups over prime fields, there is a unique $G$-invariant subgroup $U$ of rank 8 , which is generated by $w_{1} w_{9}, w_{2} w_{9}^{-1}, w_{3} w_{9}^{-1}, w_{4} w_{9}, w_{5} w_{9}, w_{6} w_{9}^{-1}, w_{7} w_{9}^{-1}$ and $w_{8}$ when $k=3$. In particular, for prime integer 3 and exponent $3^{2}$, using the NormalSubgroups command in MAGMA we can show that there is no normal subgroup of rank 8 and exponent 9 .

Next, by calculation, we can see that the subgroup $U$ is also $p$-invariant for the additional generator $p$. Hence the full automorphism group $\operatorname{GL}(2,3) \rtimes C_{2}$ can be lifted, and the covering graph is at least 2-arc-transitive.

Now we consider the lifting of $\operatorname{SL}(2,3) \rtimes C_{2}$, which is an 1-arc-regular subgroup generated by the cosets $N h$ and $N a p$ of the quotient $G_{1} / N$.

With the help of MAGMA, a reduced character table of group $S L(2,3) \rtimes C_{2}$ is given in Table 2 where $\delta$ is a primitive 3 rd root and $\phi$ is a primitive 4 th root.

Note that the traces of matrices induced by $h$, ap, hap, $(\text { haph })^{2}$ and $(h a p h)^{3}$ of orders $3,2,12,6$ and 4 , respectively, are equal to $-3,-3,1,1$ and 1 .

Table 2: The character table of the group $\operatorname{SL}(2,3) \rtimes C_{2}$

\begin{tabular}{crrrrrr}
\hline Element order & 1 & 2 & 3 & 4 & 6 & 12 \\
Class size & 1 & 6 & 4 & 6 & 4 & 4 \\
\hline$\chi_{1}$ & 1 & 1 & 1 & 1 & 1 & 1 \\
$\chi_{4}$ & 1 & -1 & $\delta$ & 1 & $-1-\delta$ & $-\delta$ \\
$\chi_{5}$ & 1 & -1 & $-1-\delta$ & 1 & $\delta$ & $1+\delta$ \\
$\chi_{7}$ & 2 & 0 & -1 & 0 & 1 & $-\phi$ \\
$\chi_{8}$ & 2 & 0 & -1 & 0 & 1 & $\phi$ \\
$\chi_{14}$ & 3 & -1 & 0 & -1 & 0 & 0 \\
\hline
\end{tabular}

Hence we can see that the character of the 9-dimensinal representation of $\operatorname{SL}(2,3) \rtimes C_{2}$ over $\mathbb{Q}$ associated with the above action of $\langle h, a p\rangle$ on $K$ is $\chi_{4}+\chi_{5}+\chi_{7}+\chi_{8}+\chi_{14}$, which is reducible to the sum of $\chi_{4}+\chi_{5}, \chi_{7}+\chi_{8}$ and $\chi_{14}$. In particular, if there exists a primitive 3rd root $\delta$, then $\chi_{4}+\chi_{5}$ is reducible to $\chi_{4}$ and $\chi_{5}$; if there exists a primitive 4 th root $\phi$, then $\chi_{7}+\chi_{8}$ is reducible to $\chi_{7}$ and $\chi_{8}$.

Therefore, we can see that for prime $k \notin\{2,3\}$, if a primitive 3 rd root $\delta$ exists, $K$ is a direct sum of four $G$-invariant subgroups of ranks 1, 1, 3 and 4; and if a primitive 4 th root $\phi$ exists, $K$ is a direct sum of four $G$-invariant subgroups of ranks $2,2,2$ and 3. (Note that, here we are only interested in the existence of $G$-invariant subgroups of rank 8.) In fact, with the help of MAGMA, if $\delta$ exists then these four $G$-invariant subgroups are generated by $\left\{w_{1} w_{2}^{\delta^{2}} w_{3}^{\delta} w_{4} w_{5}^{\delta^{2}} w_{6}^{\delta} w_{7} w_{8}^{\delta^{2}} w_{9}^{-\delta}\right\},\left\{w_{1} w_{2}^{\delta} w_{3}^{\delta^{2}} w_{4} w_{5}^{\delta} w_{6}^{\delta^{2}} w_{7} w_{8}^{\delta} w_{9}^{-\delta^{2}}\right\}$, $\left\{w_{1} w_{6}^{-1} w_{7}^{-1}, w_{2} w_{4}^{-1} w_{5} w_{6}^{-1} w_{8}^{-1}, w_{3} w_{4}^{-1} w_{9}\right\}$, and $\left\{w_{1} w_{6} w_{8}, w_{3} w_{4} w_{8}, w_{2} w_{4}^{-1} w_{5}^{-1} w_{6} w_{9}^{-1}\right.$, $\left.w_{7} w_{9}^{-1}\right\}$, respectively. Especially, by the conjugation action of generator $a$, we can see 
that a maps $w_{1} w_{2}^{\delta^{2}} w_{3}^{\delta} w_{4} w_{5}^{\delta^{2}} w_{6}^{\delta} w_{7} w_{8}^{\delta^{2}} w_{9}^{-\delta}$ to $\left(w_{1} w_{2}^{\delta} w_{3}^{\delta^{2}} w_{4} w_{5}^{\delta} w_{6}^{\delta^{2}} w_{7} w_{8}^{\delta} w_{9}^{-\delta^{2}}\right)^{\delta}$. Hence these two cyclic covers are isomorphic, and we now take them as one cover. However, no cyclic covers exist for $k \notin\{2,3\}$ when lifting the subgroup $\operatorname{GL}(2,3)$. Therefore, the cyclic covering graph is 1-arc-transitive but not 2-arc-transitive.

By [4, Proposition 2.3], this covering graph can not be 3-arc-transitive. Suppose this graph is 4-arc-transitive, then it is a cover of the Heawood graph, by [4, Proposition 3.2]. Thus the cyclic covering group must be of order $7^{e}$ for some $e$. The full automorphism group of the cyclic cover is of order $16 \cdot 8 \cdot 7^{e}$. Since the 4 -arc-transitive symmetric cubic graphs have vertex-stabilizer $S_{4}$, hence the order of cyclic covering graph is equal to $16 \cdot 8 \cdot 7^{e} / 24$ which is not an integer, contradiction. Therefore the cyclic covering graph cannot be 4-arc-transitive. Finally, again by [4, Proposition 3.4], if the covering graph is 5 -arc-transitive, then it is a cover of the Biggs-Conway graph which is of order 2352. Similar to the above argument, the covering graph cannot be 5-arc-transitive. Therefore, these cyclic covering graphs are 1-arc-transitive.

For either $k$ equal to 2 or 3 , similar to the lifting of $\mathrm{GL}(2,3)$ with the help of MAGMA, there is only one $G$-invariant subgroup of rank 8 of exponent 3 . Hence not only the subgroup $\operatorname{SL}(2,3) \rtimes C_{2}$ can be lifted but also the full automorphism group $\operatorname{Aut}(G P(8,3))$ can be lifted. In particular, by Conder's list [2] we know that there is only one symmetric cubic graph of order 48; in which case the covering graph is 2-arc-regular.

Theorem 1. Let $n=k^{e}$ be any power of a prime $k$, with $e>0$. Then the arc-transitive cyclic regular covers of the Möbius-Kantor graph with cyclic covering group of exponent $n$ are as follows:

(1) For $k \equiv 1 \bmod 3$, only the subgroup $S L(2,3) \rtimes C_{2}$ can be lifted, and there is one 1-arc-regular cover.

(2) For $k=3$ and $e=1$, there is a unique 2-arc-regular cover.

\section{Arc-transitive cyclic regular covers of the Desar- gues graph}

In this section, we classify all the arc-transitive cyclic regular covering graphs of the Desargues graph $G P(10,3)$. The automorphism group of $G P(10,3)$ is isomorphic to $S_{5} \times C_{2}$ and acts 3-arc-regularly on the arcs. There are two other 2-arc-regular subgroups $S_{5}$ and $A_{5} \times C_{2}$.

Take the group $G_{3}$, with presentation $G=\langle h, a, p, q| h^{3}=p^{2}=q^{2}=a^{2}=(q h)^{2}=$ $[p, q]=[h, p]=[a, p]=a p a q=1\rangle$. This group $G_{3}$ has a unique normal $\operatorname{subgroup~} N$ of index 240, with quotient $S_{5} \times C_{2}$.

Using the Rewrite command in MAGMA, we find that the subgroup $N$ is free of rank 11 , on generators 


$$
\begin{aligned}
& w_{1}=\text { pqahah }^{-1} a h^{-1} a h a h a h^{-1} \\
& w_{3}=\text { qhahah }^{-1} a h^{-1} \text { ahahap } \\
& w_{5}=\text { hpqahah }^{-1} a h^{-1} \text { ahahah } \\
& w_{7}=\text { pahah }^{-1} a h a h a h^{-1} a h^{-1} a h^{-1} a q \\
& w_{9}=h_{q a h a h^{-1} a h a h a h^{-1} a h^{-1} a h^{-1} a p h^{-1}} \\
& w_{11}=\left(a h a h^{-1}\right)^{5} \\
& w_{2}=p q a h^{-1} a h^{-1} a h a h a h^{-1} a h^{-1} \\
& w_{4}=q h a h^{-1} a h^{-1} a h a h a h^{-1} a p \\
& w_{6}=h p q a h^{-1} a h^{-1} a h a h a h^{-1} a h
\end{aligned}
$$

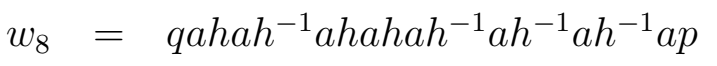

$$
\begin{aligned}
& w_{10}=\text { ahahahahahah }{ }^{-1} a h^{-1} a h^{-1} a h^{-1} a h^{-1}
\end{aligned}
$$

Easy calculations show that the generators $h, a$ and $p$ act by conjugation as below: (Note that the action of $q$ can be given by the composition apa.)

$$
\begin{aligned}
& w_{1}^{a}=w_{3} \\
& w_{2}^{a}=w_{2}^{-1} \\
& w_{1}^{h}=w_{4} \\
& w_{3}^{a}=w_{1} \\
& w_{2}^{h}=w_{3} \\
& w_{4}{ }^{a}=w_{4}{ }^{-1} \\
& w_{3}^{h}=w_{6} \\
& w_{5}^{a}=w_{7}^{-1} \\
& w_{4}^{h}=w_{5} \\
& w_{5}^{h}=w_{1} \\
& w_{6}{ }^{a}=w_{8} \\
& w_{7}^{a}=w_{5}^{-1} \\
& w_{6}{ }^{h}=w_{2} \\
& w_{8}{ }^{a}=w_{6} \\
& w_{7}^{h}=w_{5}^{-1} w_{6} w_{9}^{-1} \\
& w_{9}{ }^{a}=w_{9} w_{10} w_{11}^{-1} \\
& w_{10}{ }^{a}=w_{10}^{-1} \\
& w_{11}^{a}=w_{11}^{-1} \\
& w_{8}^{h}=w_{3} w_{4}^{-1} w_{7}^{-1} \\
& w_{1}^{p}=w_{2} \\
& w_{2}^{p}=w_{1} \\
& w_{3}^{p}=w_{4} \\
& w_{4}^{p}=w_{3} \\
& w_{5}^{p}=w_{6} \\
& w_{6}^{p}=w_{5} \\
& w_{7}^{p}=w_{7}^{-1} \\
& w_{8}^{p}=w_{8}{ }^{-1} \\
& w_{9}^{h}=w_{8} \\
& w_{9}^{p}=w_{9}^{-1} \\
& w_{10}{ }^{h}=w_{3} w_{9}^{-1} w_{10}{ }^{-1} w_{11} \\
& w_{10}^{p}=w_{8}^{-1} w_{9} w_{10} \\
& w_{11}{ }^{h}=w_{6}^{-1} w_{8} w_{10}{ }^{-1} \\
& w_{11}^{p}=w_{7} w_{9}^{-1} w_{11}
\end{aligned}
$$

Now take the quotient $G_{3} / N^{\prime}$, which is an extension of the free abelian group $N / N^{\prime} \cong$ $\mathbb{Z}^{11}$ by the group $G_{3} / N \cong S_{5} \times C_{2}$, and replace the generators $h, a, p$ and all $w_{i}$ by their images in this group. Also let $K$ denote the subgroup $N / N^{\prime}$, and let $G$ be $G_{3} / N^{\prime}$. Then, in particular, $G$ is an extension of $S_{5} \times C_{2}$ by $\mathbb{Z}^{11}$.

By the above observations, we see that the generators $h, a$ and $p$ induce linear transformations of the free abelian group $K \cong \mathbb{Z}^{11}$ as follows:

$$
h \mapsto\left(\begin{array}{rrrrrrrrrrr}
0 & 0 & 0 & 1 & 0 & 0 & 0 & 0 & 0 & 0 & 0 \\
0 & 0 & 1 & 0 & 0 & 0 & 0 & 0 & 0 & 0 & 0 \\
0 & 0 & 0 & 0 & 0 & 1 & 0 & 0 & 0 & 0 & 0 \\
0 & 0 & 0 & 0 & 1 & 0 & 0 & 0 & 0 & 0 & 0 \\
1 & 0 & 0 & 0 & 0 & 0 & 0 & 0 & 0 & 0 & 0 \\
0 & 1 & 0 & 0 & 0 & 0 & 0 & 0 & 0 & 0 & 0 \\
0 & 0 & 0 & 0 & -1 & 1 & 0 & 0 & -1 & 0 & 0 \\
0 & 0 & 1 & -1 & 0 & 0 & -1 & 0 & 0 & 0 & 0 \\
0 & 0 & 0 & 0 & 0 & 0 & 0 & 1 & 0 & 0 & 0 \\
0 & 0 & 1 & 0 & 0 & 0 & 0 & 0 & -1 & -1 & 1 \\
0 & 0 & 0 & 0 & 0 & -1 & 0 & 1 & 0 & -1 & 0
\end{array}\right),
$$




$$
a \mapsto\left(\begin{array}{rrrrrrrrrrr}
0 & 0 & 1 & 0 & 0 & 0 & 0 & 0 & 0 & 0 & 0 \\
0 & -1 & 0 & 0 & 0 & 0 & 0 & 0 & 0 & 0 & 0 \\
1 & 0 & 0 & 0 & 0 & 0 & 0 & 0 & 0 & 0 & 0 \\
0 & 0 & 0 & -1 & 0 & 0 & 0 & 0 & 0 & 0 & 0 \\
0 & 0 & 0 & 0 & 0 & 0 & -1 & 0 & 0 & 0 & 0 \\
0 & 0 & 0 & 0 & 0 & 0 & 0 & 1 & 0 & 0 & 0 \\
0 & 0 & 0 & 0 & -1 & 0 & 0 & 0 & 0 & 0 & 0 \\
0 & 0 & 0 & 0 & 0 & 1 & 0 & 0 & 0 & 0 & 0 \\
0 & 0 & 0 & 0 & 0 & 0 & 0 & 0 & 1 & 1 & -1 \\
0 & 0 & 0 & 0 & 0 & 0 & 0 & 0 & 0 & -1 & 0 \\
0 & 0 & 0 & 0 & 0 & 0 & 0 & 0 & 0 & 0 & -1
\end{array}\right),
$$

and

$$
p \mapsto\left(\begin{array}{rrrrrrrrrrr}
0 & 1 & 0 & 0 & 0 & 0 & 0 & 0 & 0 & 0 & 0 \\
1 & 0 & 0 & 0 & 0 & 0 & 0 & 0 & 0 & 0 & 0 \\
0 & 0 & 0 & 1 & 0 & 0 & 0 & 0 & 0 & 0 & 0 \\
0 & 0 & 1 & 0 & 0 & 0 & 0 & 0 & 0 & 0 & 0 \\
0 & 0 & 0 & 0 & 0 & 1 & 0 & 0 & 0 & 0 & 0 \\
0 & 0 & 0 & 0 & 1 & 0 & 0 & 0 & 0 & 0 & 0 \\
0 & 0 & 0 & 0 & 0 & 0 & -1 & 0 & 0 & 0 & 0 \\
0 & 0 & 0 & 0 & 0 & 0 & 0 & -1 & 0 & 0 & 0 \\
0 & 0 & 0 & 0 & 0 & 0 & 0 & 0 & -1 & 0 & 0 \\
0 & 0 & 0 & 0 & 0 & 0 & 0 & -1 & 1 & 1 & 0 \\
0 & 0 & 0 & 0 & 0 & 0 & 1 & 0 & -1 & 0 & 1
\end{array}\right) .
$$

These matrices generate a group isomorphic to $S_{5} \times C_{2}$, with the first two generating a subgroup isomorphic to $A_{5} \times C_{2}$; and the first and the product of the other two generating a subgroup isomorphic to $S_{5}$. Note that the matrices of orders $3,2,2,6$ and 6 representing $h, a, a p$, hap and $(h a)^{2} h^{-1} a$ have traces $-1,-3,-1,1$ and 1 , respectively.

By inspecting traces and the character tables (which can be easily given by the CharacterTable command in MAGMA) of groups $A_{5} \times C_{2}$ and $S_{5}$, we see that the 11dimensional representation of $S_{5}$ over $\mathbb{Q}$ associated with the above action of $\langle h, a p\rangle$ on $K$ is a sum of $U$ and $V$, which are two irreducible 6-dimensional and 5-dimensional representations over the rational field $\mathbb{Q}$. Also the 11-dimensional representation of $A_{5} \times C_{2}$ over $\mathbb{Q}$ associated with the action of $\langle h, a\rangle$ on $K$ is a sum of $\varphi_{1}$ and $\varphi_{2}$, which are characters of two irreducible 6-dimensional and 5-dimensional representations over the rational field $\mathbb{Q}$. However, in particular, if there exist zeros of the polynomial $t^{2}-t-1, \varphi_{1}$ is reducible to a sum of $\varphi_{1,1}$ and $\varphi_{1,2}$ each of which is a character of an irreducible 3 -dimensional representation.

Therefore, for any prime $k$ other than 2, 3 and 5 , there is no $\langle h, a p\rangle$ - and $\langle h, a\rangle$-invariant subgroup of rank 10; equivalently, no cyclic regular cover exists.

For prime $k=3$ and 5 , with the help of MAGmA, there is also no $\langle h, a p\rangle$ - and $\langle h, a\rangle$ invariant subgroup of rank 10. Thus there are no cyclic regular covers.

For prime $k=2$, with the help of MAGMA, there are only two $\langle h, a\rangle$-invariant subgroups of rank 10 of exponent 2 and 4 , respectively. Thus, correspondingly, there are two 
cyclic covering graphs of order 40 and 80 . Also there are only two $\langle h, a p\rangle$-invariant subgroups of rank 10 of exponent 2 and 4. By Conder's list [2], we know that there are unique symmetric cubic graphs of orders 40 and 80, respectively, each of which is 3-arc-regular. Hence the above two cyclic covering graphs are exactly these two graphs.

Theorem 2. There are only two arc-transitive cyclic regular covers of the Desargues graph, both are 3-arc-transitive, with cyclic covering groups $C_{2}$ and $C_{4}$, respectively.

\section{Dihedral regular covers of cubic graphs}

First of all, arc-transitive cubic graphs of small order like the complete graph $K_{4}$, the complete bipartite graph $K_{3,3}$, the 3 -cube $Q_{3}$ and the Petersen graph are well known. The arc-transitive properties of each of the above graphs are as follows.

The complete graph $K_{4}$ is 2-arc-regular with automorphism group $S_{4}$, and the only arc-transitive subgroup of automorphisms of $K_{4}$ is the subgroup $A_{4}$, which acts regularly on the arcs. The complete bipartite graph $K_{3,3}$ is 3-arc-regular. Its automorphism group is the wreath product $S_{3}>C_{2}$, and this contains three arc-transitive subgroups which act 1-, 2- and 2-arc-regularly on the arcs of $K_{3,3}$, respectively. In particular, two of these three subgroups are minimal, one is the group $A_{3} \succ C_{2}$ which acts 1-arc-regularly, while the other is $\left(A_{3} \times A_{3}\right) \rtimes C_{4}$ which acts 2-arc-regularly. The 3 -cube $Q_{3}$ is 2-arcregular, and its automorphism group is the direct product $S_{4} \times C_{2}$. And the only arctransitive proper subgroups of automorphisms are $S_{4}$ and $A_{4} \times C_{2}$, each of which acts 1-arc-regularly on the arcs of $Q_{3}$. And finally, the Petersen graph is a 3-arc-regular graph. Its automorphism group is the symmetric group $S_{5}$, and the only other arc-transitive subgroup of automorphisms is the subgroup $A_{5}$, which acts 2-arc-regularly.

Before investigating the dihedral covers, we remind readers of the following useful result given by Gardiner and Praeger in [6].

Theorem 3. [6] Let $\Gamma$ be a connected $G$-symmetric graph of valency p a prime. For each normal subgroup $N$ of $G$ one of the following holds:

(a) $\Gamma$ is $N$-symmetric;

(b) $N$ acts regularly on vertices, so $\Gamma$ is a Cayley graph for $N$;

(c) $N$ has just two orbits on vertices and $\Gamma$ is bipartite; or

(d) $N$ has $r \geqslant p+1$ orbits on vertices, the natural quotient graph $\Gamma_{N}$ on $N$-orbits is $G / N$-symmetric of valency $p$, and $\Gamma$ is a topological cover of $\Gamma_{N}$.

Now, suppose graph $\tilde{X}$ is an arc-transitive dihedral regular $D_{n}$-cover of cubic graph $X$ where dihedral group $D_{n}$ is of degree $n$ (here, we always assume $n>2$ ), then we have the following lemma:

Lemma 4. $\tilde{X}$ is a cyclic regular cover of a 2 -cover of $X$.

Proof. Since $\tilde{X}$ is an arc-transitive dihedral cover of $X$, then there exists an arc-transitive subgroup $D_{n} \rtimes A$ of $\operatorname{Aut}(\tilde{X})$ which is the lifting subgroup of an arc-transitive subgroup $A$ of $\operatorname{Aut}(X)$. Let $C_{n}$ be the cyclic subgroup of $D_{n}$, then $C_{n}$ is normal in $D_{n} \rtimes A$. Especially, 
$C_{n}$ is a semi-regular subgroup of $\operatorname{Aut}(\tilde{X})$. Thus by Theorem 3 , the quotient graph $\tilde{X} / C_{n}$ is an arc-transitive 2-cover of $X$.

For the complete graph $K_{4}$, we have the following classification of all arc-transitive dihedral regular $D_{n}$-covers.

Theorem 5. For $n \neq 2$, graph $X$ is an arc-transitive dihedral regular $D_{n}$-cover of $K_{4}$ if and only if it is an arc-transitive cyclic regular $C_{n}$-cover of the 3-cube $Q_{3}$.

Before proving the above theorem, in [3], Conder and Ma gave the following results:

Theorem 6. [3] Let $n=k^{e}$ be any power of a prime $k$, with $e>0$. Then the arctransitive cyclic regular covers of the 3 -cube $Q_{3}$ with cyclic covering groups of exponent $n$ are as follows:

(1) if $k \equiv 1 \bmod 3$, only the subgroup $A_{4} \times C_{2}$ can be lifted and there is one 1-arcregular cover.

(2) If $k=3$ and $e=1$, there is one 2-arc-regular cover.

(3) If $k=2$ and $e=1$, there is one 2-arc-regular cover.

Proof of Theorem 5: By Lemma 4, we know that $X$ is an arc-transitive cyclic cover of the $Q_{3}$ which is the only arc-transitive 2-cover of the $K_{4}$. From the above Theorem 6 , we know that there are only three types of cyclic covers. The first type, namely when $k \equiv 1 \bmod 3$, is of automorphism group $C_{n} \rtimes\left(A_{4} \times C_{2}\right)$. Note that from [3, Section 6], we know that $C_{n} \rtimes\left(A_{4} \times C_{2}\right)$ is generated by (the images of) elements $v_{t}$ and $h$, ap where $\left\langle v_{t}\right\rangle \cong C_{n}$ and $\langle h, a p\rangle \cong A_{4} \times C_{2}$. And also by [3, Page 235, Paragraph 8] we have $v_{t}{ }^{h}=v_{t}^{t}$ and $v_{t}^{a p}=v_{t}^{-1}$, hence $v_{t}^{(h a p)^{3}}=v_{t}^{-1}$ where $(h a p)^{3}$ is of order 2 and $\left\langle(h a p)^{3}\right\rangle \cong C_{2}$ which is normal in $A_{4} \times C_{2}$. Therefore we have $C_{n} \rtimes\left(A_{4} \times C_{2}\right) \cong D_{n} \rtimes A_{4}$ which suggests that $X$ is a dihedral regular cover of $K_{4}$.

For $k=3$, similarly, by [3, Page 235, Paragraph -2] the order 3 covering group is generated by $u v$, and the conjugation action of $h$ and $a p$ are $(u v)^{h}=u v$ and $(u v)^{a p}=$ $(u v)^{-1}$. Thus $(u v)^{(h a p)^{3}}=(u v)^{-1}$. Hence $C_{3} \rtimes\left(A_{4} \times C_{2}\right) \cong D_{3} \rtimes A_{4}$. (Note that the cyclic covering graph is of order 24, the structure of the automorphism group can also be easily checked by Magma.)

Remark 7. We know that there is a unique symmetric cubic graph of order 16 which is the Möbius-Kantor graph. In particular, from [3], we know that it is a cyclic $C_{4}$-cover of the complete graph $K_{4}$ and also a 2-cover of the 3-cube $Q_{3}$.

Corollary 8. Let $X$ be an arc-transitive dihedral regular $D_{n}$-cover of $K_{4}$, then $n$ is of the following possibilities:

(1) 3 or 6 ; or

(2) $2^{i} 3^{j} k^{e}$ for $i, j \in\{0,1\}$ and prime integer $k \equiv 1 \bmod 3$ and $e>0$.

Note that the the product of integers 2,3 and $k^{e}$ is just the order of each cyclic covering group which comes from the direct product of cyclic groups $C_{2}, C_{3}$ and $C_{k^{e}}$.

About the complete graph $K_{3,3}$, we have the following result. 
Theorem 9. The complete bipartite graph $K_{3,3}$ has no arc-transitive dihedral regular cover.

Proof. Suppose $K_{3,3}$ has an arc-transitive dihedral regular cover $\mathcal{D}$, then by Lemma 4 , $\mathcal{D}$ is a cyclic regular cover of a 2-cover of $K_{3,3}$. However, we know that $K_{3,3}$ has no arc-transitive 2-cover, and in fact, there is no arc-transitive cubic graph of order 12 , contradiction. Hence $K_{3,3}$ has no arc-transitive dihedral covering graph.

For the 3-cube graph $Q_{3}$, the classification of arc-transitive dihedral covers is as follows.

Theorem 10. Let $X$ be an arc-transitive dihedral regular $D_{n}$-cover of the $Q_{3}$, then $n$ is equal to 3 .

Proof. We know that each dihedral regular cover of $Q_{3}$ is a cyclic regular cover of the Möbius-Kantor graph. In Theorem 1, there are two types of cyclic regular covers of the Möbius-Kantor graph. Firstly, if there exists a primitive 3rd root $\delta$, then the cyclic covering groups of the cyclic covers are generated by $u=\left\{w_{1} w_{2}^{\delta^{2}} w_{3}^{\delta} w_{4} w_{5}^{\delta^{2}} w_{6}^{\delta} w_{7} w_{8}^{\delta^{2}} w_{9}^{-\delta}\right\}$ and $v=\left\{w_{1} w_{2}^{\delta} w_{3}^{\delta^{2}} w_{4} w_{5}^{\delta} w_{6}^{\delta^{2}} w_{7} w_{8}^{\delta} w_{9}^{-\delta^{2}}\right\}$, respectively. Since these two covering graphs are isomorphic, here we only consider the covering group generated by $u$. The images of $u$ under the conjugation actions of generators $h$ and $a p$ are $u^{4}$ and $u^{-1}$. Since $S L(2,3) \rtimes C_{2}=$ $\langle h, a p\rangle$, there is a unique normal subgroup of order 2 which is generated by $(h a p h)^{6}$. And the image of $u$ by the conjugation action of $(h a p h)^{6}$ is equal to $u^{16^{6}}$. Since $k \equiv 1 \bmod 3$ but $16^{6}+1 \equiv 2 \bmod 3$. Hence $u^{16^{6}} \neq u^{-1}$, which suggests there is no dihedral normal subgroup of $S L(2,3) \rtimes C_{2}$.

Secondly, for $k=3$ and the cyclic covering group of order 3 , the covering graph is 2-arc-regular and of order 48. With the help of MAGMA, we can easily verify that its a dihedral regular covering graph of the $Q_{3}$, with automorphism group isomorphic to $D_{3} \rtimes\left(S_{4} \times C_{2}\right)$.

In [7], the author classified all the arc-transitive cyclic covers of the dodecahedron graph, and gave the following result.

Theorem 11. [7] Let $n=k^{\ell}$ be any power of a prime $k$, with $\ell>0$. Then the arctransitive cyclic regular covers of the dodecahedron graph with covering group of exponent $n$ are as follows:

(a) If $k=2$, there are exactly two such covers, namely

- one 3 -arc-transitive cover with covering group $\mathbb{Z}_{2}$ where $\ell=1$,

- one 3-arc-transitive cover with covering group $\mathbb{Z}_{4}$ where $\ell=2$.

(b) If $k=3$, there is exactly one such cover, namely

- one 2 -arc-transitive cover with covering group $\mathbb{Z}_{3}$ where $\ell=1$.

(c) There is no arc-transitive cyclic cover for other prime integer $k \neq 2,3$.

Corollary 12. All the arc-transitive cyclic regular covering graphs of the Desargues graph are also arc-transitive cyclic regular covers of the dodecahedron graph. 
Now, we can give the following results for arc-transitive dihedral regular $D_{n}$-covers of the Petersen graph.

Theorem 13. Let $X$ be an arc-transitive dihedral regular $D_{n}$-cover of the Petersen graph, then $n$ is equal to either 3 or 6.

Proof. First of all, every dihedral regular cover of the Petersen graph is a cyclic regular cover of a 2-cover of the Petersen graph. And we know that there are two 2-covers of the Petersen graph which are the dodecahedron graph and the Desargues graph. However by Corollary 12, we only need to consider the cyclic covers of the dodecahedron graph.

By Theorem 11, we know that there are only finitely many cyclic covers. For $n=2$, by [3], we know that the Petersen graph has a $\left(C_{2}\right)^{2}$-cover. For $n=4$, the covering graph is of order 80 with automorphism group isomorphic to $Q_{8} \rtimes S_{5}$ where $Q_{8}$ is the quaternion group of order 8 . Hence its a 'quaternion' $Q_{8}$-cover of the Petersen graph instead of a dihedral cover.

Similarly, for $n=3$, we have a 2-arc-transitive $C_{3}$-covering graph of automorphism group $C_{3} \rtimes\left(A_{5} \times C_{2}\right)$. With the help of MAGMA, we have $C_{3} \rtimes\left(A_{5} \times C_{2}\right) \cong D_{3} \rtimes A_{5}$ which suggests that its a dihedral regular $D_{3}$-cover of the Petersen graph.

Therefore, the Petersen graph only has two dihedral covers with covering groups $D_{3}$ and $D_{6}$.

\section{Acknowledgements}

The author is indebted to the referee for the valuable comments and suggestions. And the author acknowledges the use of the MAGMA computational package [1], which helped show many of the results given in this paper.

\section{References}

[1] W. Bosma, J. Cannon and C. Playoust. The Magma Algebra System I: The User Language. J. Symbolic Comput., 24:235-265, 1997.

[2] M. D. E. Conder. Trivalent (cubic) symmetric graphs on up to 10000 vertices. http://www. math. auckland.ac.nz/ conder/symmcubic10000list.txt, 2012.

[3] M. D. E. Conder and J. Ma. Arc-transitive abelian covers of cubic graphs. J. Algebra, 387:215-242, 2013.

[4] M. D. E. Conder and R. Nedela. A refined classification of symmetric cubic graphs. J. Algebra, 322:722-740, 2009.

[5] D. Ž. Djoković and G. L. Miller. Regular groups of automorphisms of cubic graphs. J. Combin. Theory Ser. B, 29:195-230, 1980.

[6] A. Gardiner and C. E. Praeger. On 4-valent symmetric graphs. Europ. J. Combin., 15:375-381, 1994.

[7] J. Ma. On 3-arc-transitive covers of the Dodecahedron graph. preprint. 
[8] J. Ma, Arc-transitive abelian regular covering graphs. preprint.

[9] A. Malnič, D. Marušič and P. Potočnik. Elementary abelian covers of graphs. J. Algebraic Combin., 20:71-97, 2004. 